\title{
7
}

\section{THE PHYSICAL BASIS OF THE POLARIMETRIC METHOD FOR DERIVING ASTEROID ALBEDOS}

\author{
T. GEHRELS
}

Physical interpretations of negative polarization and opposition effect confirm the existence of a surface layer on the asteroids of their own dust. Future work should establish whether the impacting grains are cometary or asteroidal/meteoritic.

Reflectivity determination of asteroids has become a major tool for leaming the composition and for studying the interrelations with meteorites. The reflectivity determination is presently done by radiometric and polarimetric methods - interferometry and speckle imaging have not as yet been applied to asteroids - and their results are in fair agrecmont with each other (see Morrison's chapter in this book). The radiometric method is, however, believed to be more direct and better based on physical concepts. It is the purpose of this note to summarize the physical cxplanation of the polarimetric method. At the end it will be confirmed that the asteroids have a dusty surface which is presumably due to impacts of interplanetary particles.

The polarimetric method is, in fact, based on physical concepts as well as are the phenomena of color and reflectivity of fabrics and surfaces.

Let us consider separately the light singly reflected by the surface (or by the ensemble of surfaces of particles on the asteroid), and the light that is multiply scattered among the particles. Surface reflection in physical optics follows Fresnel's Law of which the shape of the relation of 1 inear and positive polarization is well known: zero at zero phase, a maximum near Brewster's angle $\left(\sim 110^{\circ}\right)$, and zero polarization again at $180^{\circ}$. Superposed on that is the multiple scattering by the particles, which is relatively important near zero phase when the Fresnel polarization is low. That the multiple-scattering polarization is negative (i.e., with electric vector maximum in the scattering plane through Sun, asteroid and observer) is seen with a sketch as in Fig. 1.

Figure 1 is a view straight down into a flat-bottom pit while the sunlight comes in slightly from the left, at small phase angle. First we consider the multiple reflection from $B$ to $A$ to the observer. The light received at $B$ is strongly polarized by Fresnel reflection. The electric vector maximum is paraliel to the scattering plane and so it is after scattering at $A$ towards the observer, i.e., negative polarization. Scattering via $C$ and $A$ gives positive polarization, but micro-shadowing within the texture limits this flux. 


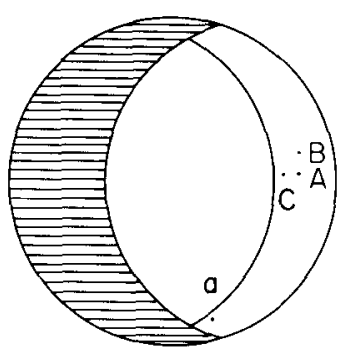

Figure 1. A sketch of a cylindrical pit in an asteroidai surface. The point $\underline{C}$ is deeper down the illuminated wall than $\underline{A}$ and $\underline{B}$ are; the opposite face of the pit is in the (hatched) shadow.

Similarly at the flat bottom of this pit, there will be a little more negative than positive polarization, because of shadowing, and on the wall also there is a greater area of negative polarization than of positive (positive near a, for instance, in Fig. 1). Within $\sim 7^{\circ}$ of opposition most of the shadowing disappears as we look into the porous texture ("opposition effect"), but now symmetry and reciprocity are reached that cancel polarizations.

The "negative" and "Fresnel" polarizations combine into a phase dependence as follows. At very small phase angles, dusty asteroids (see below) must at first have some negative polarization by the above mechanism. Since the amount of negative polarization is smal1, while the Fresnel polarization comes in strongly with increasing phase, there must occur, as the phase angle increases (see Fig. 2): a minimum in the polarization $\left(P_{\min }\right)$, a rising branch, zero polarization (at the inversion phase angle), and a maximum of the polarization $\left(P_{\max }\right)$, followed by the Fresnel decline towards crescent phases.

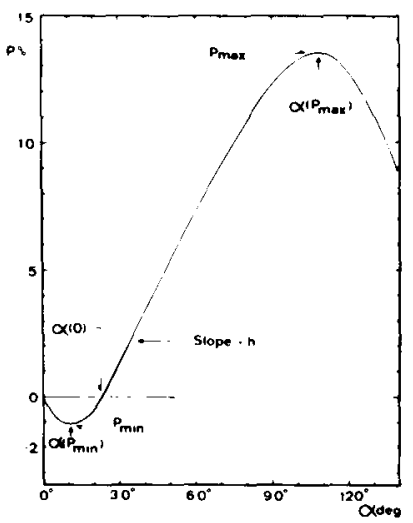

E'igure 2. Parameters for polarization-phase curves: $\alpha$ is the solar phase angle; the polarization is given in percentages in the ordinates; a $(0)$ is the phase angle of the inversion point.

The Fresnel part of the polarization can be expressed by

$$
P_{F}=\frac{R_{F}}{R} \frac{B_{1}-B_{2}}{B_{1}+B_{2}},
$$


where the Fresnel reflectivity $R_{F}=(m-1)^{1} /(m+1)^{2}$ for refractive index $m, R$ is the observed reflectivity at normal incidence, $B_{1}=\sin ^{2}(\underline{i-r}) / \sin ^{2}(\underline{i}+r)$ and $B_{2}=\tan ^{2}(\underline{i}-\underline{r}) / \tan ^{2}(\underline{i}+\underline{r})$ with $\underline{i}$ being the angle of incidence and $\sin \underline{r}=$ (sin $\mathrm{i}) / \mathrm{m}$. To a first approximation $\mathrm{i}=1 / 2 \alpha$ where $\alpha$ is the phase angle.

The above expression incorporate $\bar{s}$ the "Umov Effect," that dark surfaces polarize more than bright ones in direct proportion to their reflectivity (Bowell and Zellner 1974). To a good approximation the rising branch is linear in the phase-angle range $20^{\circ}<|\alpha|<40^{\circ}$ (Gehrels 1970). The first application of the polarimetric method for the determination of asteroid albedos was for Icarus by Gehrels et al. (1970).

A numerical analysis of the above polarization phenomena has been made by Wolff (1975). He shows that a negative polarization branch is indeed obtained at small phases, by double Fresnel reflection and shadows to spoil the symmetry as described above. By adding in a mixture of unpolarized, diffusely scattered light due to edge diffraction, high-order scattering, etc., he generates polarization-phase curves much like those of the moon and the asteroids. I believe that more theoretical work could be done, for example to derive an explicit theoretical form of the slope-albedo law. Qualitatively one can see that this relation is non-linear, because of the onset of higher-order scattering (very bright objects) and absorption (very dark objects), diminishing $P_{\max }$ and raising $\mathrm{P}_{\mathrm{min}}$. The theoretical fit would have to take wavelength dependence into account (Zellner and Gradie 1976). The radiometric method needs further work as well because its model contains assumptions that may affect the albedo determination (Hansen 1977, Morrison 1977).

The second-order scattering that I refer to above (with Fig. 1) is substantiated by Dollfus(1956) in an experiment which produced negative polarization for a powdered surface sample, while the negative polarization disappeared when these same dust particles were separated widely (when falling through the air).

The presence of a porous dust layer on the asteroids seems, in fact, proven by these interpretations and Wolff's analysis of the polarization-phase curve and the opposition effect. The opposition effect (i.e., the nonlinear brightness increase from phase angles $\sim 7^{\circ} \rightarrow 0^{\circ}$ ) is remarkably the same for the 5 asteroids that have been observed with sufficient precision to date: Ceres, Vesta, Hebe, Massalia, and Lydia (Gehrels and Taylor 1977). Impacting interplanetary grains apparently make on all five about the same porous-dust layer that is needed for the interpretation of the opposition effect and negative polarization. However, the impacting particles must remove more material than they deliver because these asteroids have their own compositional class. Their phase factors (i.e., brightness change in magnitudes per degree phase angle; $70<|a|<25^{\circ}$ ) also differ (Gehrels and Taylor 1977): Ceres (C-class, 0.036 $\mathrm{mag} / \mathrm{deg})$; Vesta $(\mathrm{U}, 0.0253)$; Hebe (S, 0.0266); Massalia (S, 0.031); and Lydia (C, 0.032). Systematically different phase factors for asteroids of different compositional classes are reported by Bowell (1977).

The small asteroids of the Palomar-Leiden Survey have a large phase factor and pernaps also a steeper opposition effect than the bright ones (van Houten 1971). From Bowe1l's results it would appear that these differences are due to differences in composition: as we observe fainter asteroids, the predominance of $\mathrm{C}$ objects, that are farther from the sun, increases. One would also expect the C-type asteroids to have softer structures than the $S$ - and other types, to be more peppered into fairy-castle structures, and to yeld a stronger opposition effect; the above observations of Ceres and Lydia, do not confirm this idea, however. Van Houten's primary conclusion is that the Trojans differ from the asteroids, by having a much weaker opposition effect which indicates a different surface texture; would the Trojan material be harder?

Asteroid (16) Psyche did not seem to have an opposition effect in 1974 (Taylor et al. 1976). This is probably due to aspect variation: the precise orientation in space of an asteroid with irregular shape ( 3 or more axes) must 


\section{GEHRELS}

be taken into account in order to obtain their proper phase relation (Sather 1976). If this is done, with future observations, and if an asteroid is found to have no opposition effect, it would be the first discovery of a rock withou an appreciable layer of dust on the surface.

These topics are important for future study of the interrelations of asteroids, comets and meteorites. Pioneers 10 and 11 observe particles without signature of the asteroid belt, out to $5 \mathrm{AU}$, that therefore probably are cometary (Humes 1976; also see p. 559 of that book). Just as they impact the meteoroid detectors these particles will impact the asteroids, and they are strong enough to penetrate the detector walls. The dusty surface structures therefore may be due to cometary impact particles, but further observations of opposition effect and negative polarization, for various asteroids at various distances from the sun, are needed.

\section{REFERENCES}

Bowe11, E. 1977, Bull. Am. Astron. Soc., in press.

Bowel1, E., and Zellner, B. 1974, Planets, stars and Nebulae, Studied with Photopolarimetry, ed. T. Gehrels (Tucson, Arizona: University of Arizona Press), p. 384 .

Dollfus, A. 1956, Ann. Astrophys., 19, 83.

Gehrels, T. 1970, Surfaces and Interiors of Planets and Satellites, ed. A. Dollfus (New York: Academic Press), p. 354.

Gehrels, T., and Taylor, R. C. 1977, Astron. J., 82, in press.

Gehre1s, T., Roemer, E., Taylor, R. C., and Zellner, B. H. 1970, Astron. J., $75,193$.

Hansen, O. L. 1977, Icarus, in press.

Humes, D. H. 1976, Jupiter, ed. T. Gehrels (Tucson: Univ. of Ariz. Press), P. 1052 .

Morrison, D. 1977, Icarus, in press.

Sather, R. E. 1976, Astron. J., 81, 67 .

Taylor, R. C., Gehrels, T., and Capen, R. C. 1976, Astron. J., 81, 780.

van Houter, C. J. 1971, Physical Studies of Minor Planets, ed. T. Gehrels (Wash., D. C.: National Aeronautics and Space Administration), NASA $\mathrm{SP}-267, \mathrm{p} .185$.

Wolff, M. 1975, Applied Optics, 14, 1395.

Zellner, B., and Gradje, J. 1976, Astron. J., 81, 262. 